\title{
Bilateral Pheochromocytoma with Hypertrophic Obstructive Cardiomyopathy: A Rare Case
}

\author{
${ }^{1}$ Brajesh B Gupta, ${ }^{2}$ Sanjay S Changole, ${ }^{3}$ Vishal Nandagawali, ${ }^{4}$ Chandratej Chandrkant Kadam
}

\begin{abstract}
Pheochromocytomas are catecholamine-secreting tumors of neuroectodermal origin. Different clinical presentations, various preoperative and intraoperative complications and their potential to become malignant provide a challenge in diagnosing and managing these cases. We report a rare case of bilateral pheochromocytoma with hypertrophic obstructive cardiomyopathy. He was diagnosed with bilateral pheochromocytoma with hypertrophic obstructive cardiomyopathy presenting at the age of 15 years without evidence of multiple endocrine neoplasia type 2 (MEN-2), or any familial syndrome or metastasis and then offered a surgery which was first cancelled due to uncontrolled hypertension. He was then taking alpha blocker for 8 years until he was again admitted to our institute. After prior blood pressure control with alpha and beta blocker drugs, open bilateral adrenalectomy was done without any complications. Intraoperative spikes in blood pressure was managed with short acting antihypertensives, such as esmolol and labetalol. Postoperatively, patient is given steroid and under follow-up to rule out malignancy and other complications.
\end{abstract}

Keywords: Bilateral pheochromocytoma, Hypertrophic obstructive cardiomyopathy, Bilateral adrenalectomy.

How to cite this article: Gupta BB, Changole SS, Nandagawali V, Kadam CC. Bilateral Pheochromocytoma with Hypertrophic Obstructive Cardiomyopathy: A Rare Case. World J Endoc Surg 2014;6(3):107-109.

Source of support: Nil

Conflict of interest: None

\section{INTRODUCTION}

Pheochromocytomas are catecholamine-secreting tumors of neuroectodermal origin. Pheochromocytoma is found in about 0.1 to $0.6 \%$ of hypertensive patient. ${ }^{1}$ Approximately, $85 \%$ of these arise from the adrenal medulla and $15 \%$ arise from chromaffin tissue in extraadrenal sites which are referred as paragangliomas. Incidence of bilateral pheochromocytoma is about $10 \%$ of all pheochromocytomas. $<90 \%$ of pheochromocytoma are sporadic, however in about $10 \%$ of cases it is associated with the familial syndromes such multiple endocrine

\footnotetext{
${ }^{1}$ Professor, ${ }^{2}$ Associate Professor, ${ }^{3}$ Lecturer, ${ }^{4}$ Resident

${ }^{1-4}$ Department of General Surgery, Government Medical College, Nagpur, Maharashtra, India

Corresponding Author: Chandratej Chandrkant Kadam Resident, Department of General Surgery, Government Medical College, Nagpur, Maharashtra, India, Phone: 9028144140 e-mail: chandratejkadam@gmail.com
}

neoplasia type 2 (MEN-2), Von Hippel-Lindau (VHL) syndrome, neurofibromatosis type 1 (NF-1), and familial paragangliomas. Thus, screening of high risk individuals to find out specific germ line mutation of these syndromes becomes important. ${ }^{1}$ Different clinical presentations, various preoperative and intraoperative complications and their potential to become malignant provide a challenge in diagnosing and managing these cases. Good clinical evaluation, biochemical confirmation, tumor localization and excision of the tumor after prior stabilization with alpha or beta blockers offer a chance of cure for these rare but lethal tumors. Though hypertension, sweating and palpitations are the presenting symptoms in most of the cases, rare cases may present with unusual complications, such as catecholamine induced hypertrophic or dilated cardiomyopathy, arrhythmias. ${ }^{2}$ Here, we report a rare case of bilateral pheochromocytoma with hypertrophic obstructive cardiomyopathy.

\section{CASE REPORT}

We report a case of 23-year-old male presenting with chief complaints of episodes of palpitations off and on associated with headache, sweating since 10 days. He had history of similar episodes since the age of 15 years when he was first evaluated and found to have raised blood pressure of 230/120 mm Hg with pulse rate of 130/min. He was further evaluated by doing ultrasonography (USG) of abdomen and found to have bilateral suprarenal masses suggestive of bilateral adrenal tumor. Patient was further evaluated at higher center by doing biochemical evaluation including urinary metanephrines, CT scan of abdomen, 2D echo and metaiodobenzylguanidine (MIBG) scan and was diagnosed as case of bilateral pheochromocytoma with hypertrophic obstructive cardiomyopathy (HOCM). Patient was offered surgery but was postponed of high BP on operating table. Patient was discharged on alpha blocker Prazosin in twise daily (BID) doses. Patient was taking alpha blocker since then for 8 years and was having intermittent episodes of sweating, palpitation, headache which suddenly got aggravated 10 days back when patient came to our institute again. He also complained of mild abdominal discomfort.

He was evaluated for the same. He still had classical 'Pheochromocytoma paroxysms' with highest BP of 220/130 mm Hg and PR of 124/min. Patient did not has family history of similar complaints in parents as well as 
in his one brother and one sister. There was no evidence of neurofibromatosis or retinal hemangioma. Fundus examination was reported as normal. Routine blood and urine investigations were normal. Post meal blood sugar was $168 \mathrm{mg} / \mathrm{dl}$. Electrocardiogram (ECG) showed left ventricular hypertrophy. Chest X-ray was reported as normal.

Urinary 24 hours metanephrines: $4.30 \mathrm{mg} / 24 \mathrm{hr}$ (high), normal: 0 to $1.0 \mathrm{mg} / 24 \mathrm{hr}$.

Urinary 24 hours VMA: $37.90 \mathrm{mg} / 24 \mathrm{hr}$, normal: 0.00 to $13.60 \mathrm{mg} / 24 \mathrm{hr}$.

\section{CT ABDOMEN}

Computed tomography (CT) scan abdomen showed relatively well-defined supra renal masses of sizes as mentioned in Figure 1 not separately visualized from either adrenal gland with features s/o neoplastic etiology.

MIBG scan: Abnormal uptake in both supra renal regions corresponding to the masses seen in CT scan. There was no evidence of extra-adrenal uptake of the metaiodobenzylguanidine (MIBG).

Two-dimensional echocardiography: It showed nonobstructive hypertrophic cardiomyopathy with mild SAM gradient of $37.6 \mathrm{~mm} \mathrm{Hg}$ across LVOT with mild MR as shown in Figure 2.

Films of 2D Echo: Showing hypertrophic cardiomyopathy with SAM (Fig. 2).

Coronary angiography: Normal coronary and renal arteries serum basal cortisol, thyroid profiles were normal.

Serum calcitonin: $8.5 \mathrm{pg} / \mathrm{ml} \mathrm{N}$ : 5 to $18.2 \mathrm{pg} / \mathrm{ml}$

Ionic calcium: $1.1 \mathrm{mmol} / 1 \mathrm{~N}$ : 1.1 to $1.32 \mathrm{mmol} / \mathrm{l}$, total calcium: $2.1 \mathrm{mmol} / 1, \mathrm{~N}: 2.1$ to $2.6 \mathrm{mmol} / 1$.

Basal S insulin: $5.1 \mathrm{mIU} / \mathrm{ml} \mathrm{N}$ : 5-25 mIU/ml, FBS: $98 \mathrm{mg} / \mathrm{dl}$.

On the basis of above finding patient was diagnosed as case of bilateral pheochromocytoma with hypertrophic obstructive cardiomyopathy presenting at the age of 15 years without evidence of MEN-2, or any familial syndrome or metastasis. Patient was started on alpha blocker Phenoxybenzamine $10 \mathrm{mg}$ twice day, amlodipine $5 \mathrm{mg}$ twice day and was given beta blocker for 2 weeks after 4 weeks of phenoxybenzamine. After preoperative evaluation, patient was posted for bilateral adrenalectomy. Patient was adequately hydrated prior to the procedure. He was given 4 pints of $0.9 \%$ normal saline. After taking bilateral subcostal incisions first left and then right total adrenalectomy were done. Tumor on right side measured $6 \times 5 \times 3 \mathrm{~cm}$ and on left side measured $6 \times 6 \times 4 \mathrm{~cm}$ (Fig. 4 ), weighing about $60 \mathrm{gm}$ (right) and $80 \mathrm{gm}$ (left) respectively. And were sent for histopathological examination.

Intraoperative course: There was sudden rise in heart rate and the blood pressure to $228 / 130 \mathrm{~mm} \mathrm{Hg}$ after handling the tumor. This was managed by short acting drugs, such as IV esmolol bolus, drip and IV labetalol bolus. Postoperative blood pressure was about
88/50 mm $\mathrm{Hg}$ and was managed with IV fluids and IV mephentine, nor adrenaline and dopamine drips which was tapered over one day. He was also given injection hydrocortisone $100 \mathrm{mg} 6$ hourly on day one which was tapered over period of 5 days along with fludrocortisone $100 \mathrm{mcg} /$ day. On 6th day, he was started on tablet. Fludrocortisone $0.1 \mathrm{mg} /$ day and $\mathrm{T}$ prednisolone $5 \mathrm{mg}$ bid. To be taken lifelong. Patient is also counselled for 'Steroid cover under stress' (intraoperative photographs as shown in Fig. 3).

Histopathological report as shown in Figure 5 was confirmative of bilateral pheochromocytoma.

\section{DISCUSSION}

Pheochromocytoma is an adrenal medullary tumor made up of chromaffin cells and has capacity to secrete epinephrine, nor epinephrine and dopamine. The classic presenting feature of pheochromocytoma, seen in about $>90 \%$ of patients is 'paroxysm' consisting of sudden onset of headache, sweating, palpitation and anxiety associated with pallor lasting for 30 to 45 minutes. ${ }^{3,4}$ These episodes increase in frequency duration, severity as the disease

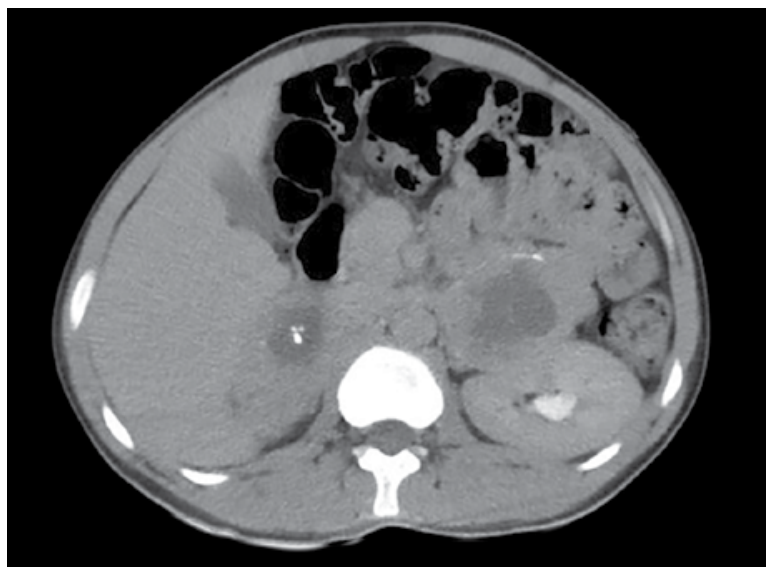

Fig. 1: CT scan showing two relatively well-defined mass lesions noted in bilateral suprarenal region of approximate size $6.5 \times 4.3 \times 6.9 \mathrm{~cm}$ on left side and $6.3 \times 3.5 \times 6.6 \mathrm{~cm}$ on right side in transverse, anteroposterior and craniocaudal axis
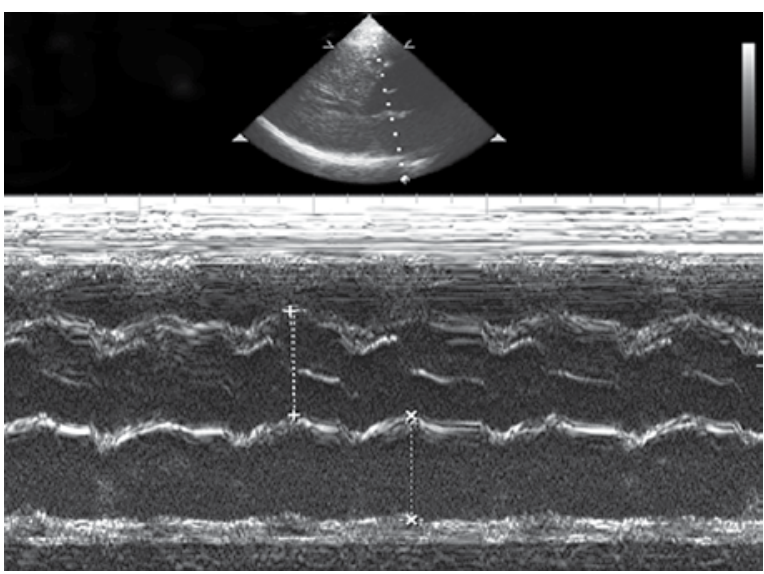

Fig. 2: Two-dimensional echo showing hypertrophic cardiomyopathy with SAM 


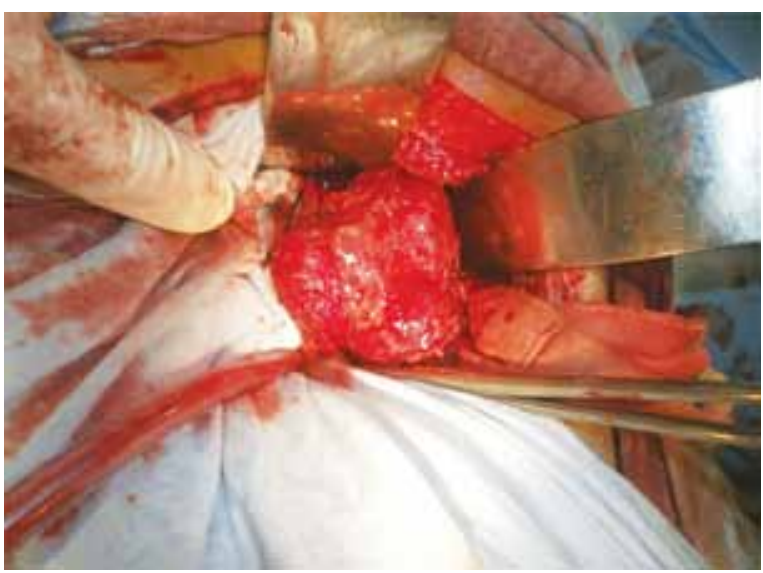

Fig. 3: During dissection of left adrenal gland (intraoperative photograph)

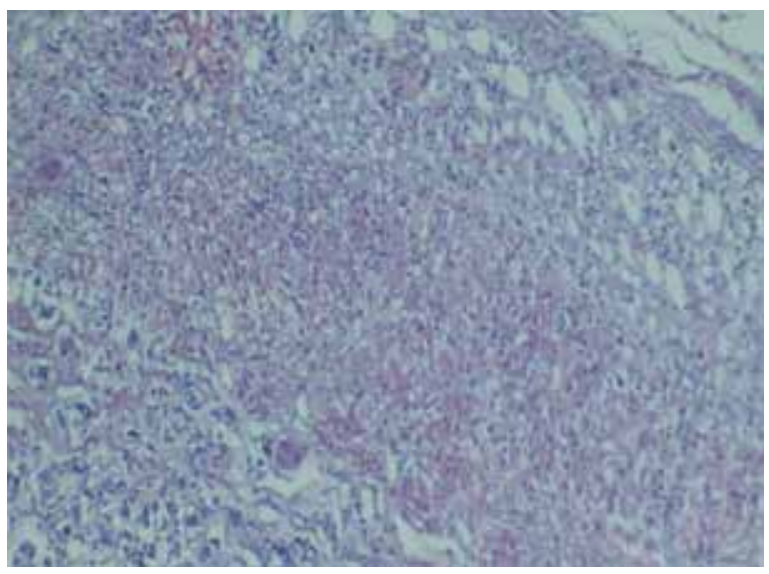

Fig. 5: Microscopic view of adrenal gland in pheochromocytoma (histopathological photograph)

progresses further. Cardiac manifestation of pheochromocytoma includes dilated or obstructive catecholamine induced cardiomyopathy, cardiogenic and noncardiogenic pulmonary edema. Thirty percent of pheochromocytoma patients show different catecholamine induced cardiomyopathy, HOCM is the rarest among all. ${ }^{5,6}$

Thus, HOCM with bilateral pheochromocytoma accounts to be the rarest and challenging case to manage both pre and intraoperatively. As use of vasodilator for pheochromocytoma can lead to sudden collapse in case of HOCM. Hence, careful use of preoperative medication, such as alpha blocker ( $\mathrm{T}$ phenoxybenzamine) and beta blocker for stabilization and intraoperative use of short acting drugs, such as esmolol and labetalol is required and use of vasodilators, such as injection sodium nitroprusside is to be avoided which is followed in this case. Perioperative morbidity and mortality in patients with pheochromocytoma associated with HOCM were 23.6 and $2.4 \%$ respectively. ${ }^{7}$ It can be reduced by preoperative planning and perioperative monitoring.

Our patient was diagnosed with this rare entity of bilateral pheochromocytoma with HOCM without any familial syndrome at the age of 15 years but due to uncontrolled blood pressure his surgery was postponed

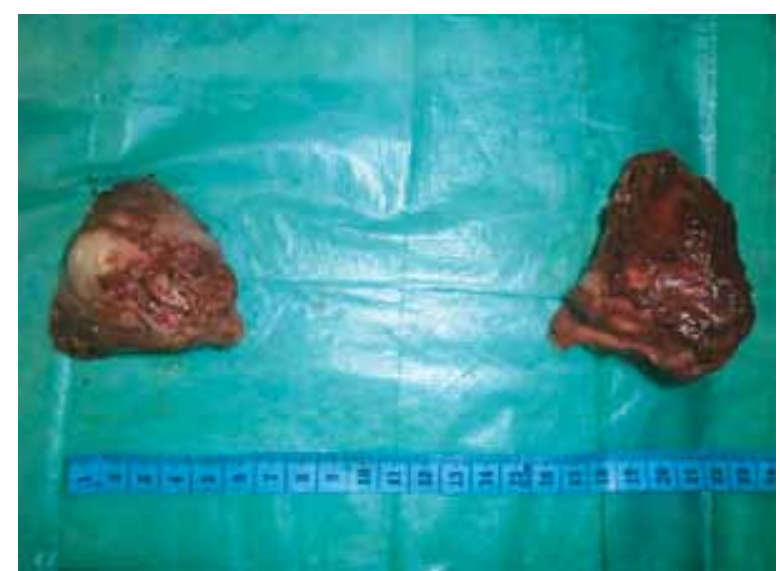

Fig. 4: Both resected adrenal specimens (specimen photograph)

and was on antihypertensive medication for about 8 years until he underwent open bilateral adrenalectomy at our institute in January 2014. At present, he is under 3 monthly follow-up with normal BP. Long-term follow-up is essential to rule out the malignant nature of tumor as histopathologically it is not possible to predict true malignant potential of tumor.

\section{CONCLUSION}

There are very few reported cases of bilateral pheochromocytoma associated with HOCM according to our literature review. ${ }^{2,8}$ This case stresses upon the importance of complete evaluation of any case of pheochromocytoma which can help in the pre and intraoperative management of associated conditions, such as HOCM which otherwise may prove to be fatal.

\section{REFERENCES}

1. Lenders JWM, Eisenhofer G, Mannelli M, Pacak K. Phaeochromocytoma. Lancet 2005;366:665-675.

2. Kassim TA, Clarke DD, Mai VQ, Mohamed Shakir KM. Catecholamine-induced cardiomyopathy. Endocr Pract 2008;14:1137-1149.

3. Manger WM, Gifford RW. Clinical and experimental pheochromocytoma. N Engl J Med 1997;336:1682-1683.

4. Bravo EL, Tagle R. Pheochromocytoma: state-of-the-art and future prospects. Endocrine Reviews 2003;24:539-553.

5. G Ilsanz FJ, Luengo C, Conejero P, Peral P, Avello F. Cardiomyopathy and phaeochromocytoma. Anaesthesia 1983;38: 888-891.

6. Park JH, Kim KS, Sul JY, et al. Prevalence and patterns of left ventricular dysfunction in patients with pheochromocytoma. J Cardiovasc Ultrasound 2011;19:76-82.

7. Plouin PF, Duclos JM, Soppelsa F, et al. Factors associated with perioperative morbidity and mortality in patients with pheochromocytoma: analysis of 165 operations at a single center. J Clin Endocrinol Metab 2001;86:1480-1486.

8. Huddle KR, Kalliatakis B, Skoularigis J. Pheochromocytoma associated with clinical and echocardiographic features simulating hypertrophic obstructive cardiomyopathy. Chest 1996;109:1394-1397. 